\title{
Anomaly of Optical Phonon in Monolayer Graphene
}

\author{
Tsuneya ANDO \\ Department of Physics, Tokyo Institute of Technology \\ 2-12-1 Ookayama, Meguro-ku, Tokyo 152-8551
}

The frequency shift and broadening of long-wavelength optical phonons due to interactions with electrons are calculated in a monolayer graphene as a function of the electron density. The broadening disappears and the frequency shift exhibits a logarithmic singularity when the Fermi energy is half of the energy of the optical phonon. The shift increases in proportion to the Fermi energy for sufficiently high electron density.

Keywords: two-dimensional graphite, electron-phonon interaction, optical phonon, broadening

\section{$\S 1$. Introduction}

In an effective-mass approximation, an electron in a graphite monolayer is described by Weyl's equation for a massless neutrino. $\left.{ }^{1,2}\right)$ Transport properties in such an exotic electronic structure are quite intriguing, and the conductivity with/without a magnetic field including the Hall effect ${ }^{3,4}$ ) and the dynamical transport ${ }^{5)}$ were investigated theoretically. The results show that the system exhibits various characteristic behaviors different from conventional two-dimensional systems. ${ }^{6)}$ Quite recently, this single layer graphite was fabricated, ${ }^{7)}$ and the magnetotransport was measured including the integer quantum Hall effect, demonstrating the validity of the neutrino description of the electronic states. ${ }^{8,9)}$

The graphene is quite unique because the electron or hole concentration in a single sheet is controlled by a gate voltage for a wide range and therefore provides a system where the phonon spectrum can be modified directly by the gate voltage. Further, the phonon frequencies near the $\Gamma$ point can be measured directly by the Raman scattering. ${ }^{10,11)}$ Quite recently effects of electron-phonon interactions on the dispersion and the broadening of optical phonons were investigated in the undoped case. ${ }^{12,13)}$ In this paper we shall study interaction effects on longwavelength optical phonons as a function of the electron concentration.

In a previous work, a continuum model suitable for a correct description of long-wavelength acoustic phonons was constructed. ${ }^{14)}$ A similar continuum model was developed for optical phonons and the Hamiltonian for electron-phonon interactions was derived also. ${ }^{15)} \mathrm{We}$ shall use this continuum model to calculate the selfenergy of phonon Green's function in a graphene. The real part of the self-energy gives an energy shift and the imaginary part provides a lifetime. In $\S 2$, the $\boldsymbol{k} \cdot \boldsymbol{p}$ scheme for the description of electronic states and a continuum model of optical phonos are reviewed very briefly. The phonon Green's function is calculated and shifts and broadening of phonon modes are discussed in $\S 3$. The results are discussed in $\S 4$ and a short summary is given in $\S 5$.

\section{$\S 2$. Formulation}

\subsection{Effective-mass description}

In a graphite sheet the conduction and valence bands consisting of $\pi$ orbitals cross at $\mathrm{K}$ and $\mathrm{K}^{\prime}$ points of the Brillouin zone, where the Fermi level is located. ${ }^{16,17)}$ Electronic states of the $\pi$-bands near a $\mathrm{K}$ point are described by the $\boldsymbol{k} \cdot \boldsymbol{p}$ equation: ${ }^{1-2)}$

$$
\mathcal{H}_{0} \boldsymbol{F}(\boldsymbol{r})=\varepsilon \boldsymbol{F}(\boldsymbol{r}),
$$

with

$$
\mathcal{H}_{0}=\gamma\left(\begin{array}{cc}
0 & \hat{k}_{x}-\mathrm{i} k_{y} \\
\hat{k}_{x}+\mathrm{i} \hat{k}_{y} & 0
\end{array}\right)=\gamma(\boldsymbol{\sigma} \cdot \boldsymbol{k}),
$$

where $\gamma$ is a band parameter, $\boldsymbol{\sigma}=\left(\sigma_{x}, \sigma_{y}\right)$ is the Pauli spin matrix, and $\hat{\boldsymbol{k}}=\left(\hat{k}_{x}, \hat{k}_{y}\right)=-\mathrm{i} \boldsymbol{\nabla}$ is a wave-vector operator.

The wave function is written as

$$
\boldsymbol{F}_{s \boldsymbol{k}}(\boldsymbol{r})=\frac{1}{L} \boldsymbol{F}_{s, \boldsymbol{k}} \exp (\mathrm{i} \boldsymbol{k} \cdot \boldsymbol{r}),
$$

with

$$
\boldsymbol{F}_{s, \boldsymbol{k}}=\frac{1}{\sqrt{2}}\left(\begin{array}{c}
s \\
\mathrm{e}^{\mathrm{i} \theta(\boldsymbol{k})}
\end{array}\right),
$$

where $L^{2}$ is the area of the system, $s=+1$ and -1 denote the conduction and valence bands, respectively, and

$$
k_{x}=k \cos \theta(\boldsymbol{k}), \quad k_{y}=k \sin \theta(\boldsymbol{k}), \quad k=|\boldsymbol{k}| .
$$

The corresponding energy is given by

$$
\varepsilon_{s}(\boldsymbol{k})=s \varepsilon(\boldsymbol{k}),
$$

with

$$
\varepsilon(\boldsymbol{k})=\gamma k \text {. }
$$

For the $\mathrm{K}^{\prime}$ point, the Hamiltonian is given by

$$
\mathcal{H}_{0}=\gamma\left(\begin{array}{cc}
0 & \hat{k}_{x}+\mathrm{i} \hat{k}_{y} \\
\hat{k}_{x}-\mathrm{i} \hat{k}_{y} & 0
\end{array}\right)=\gamma\left(\boldsymbol{\sigma}^{*} \cdot \hat{\boldsymbol{k}}\right),
$$

and the corresponding wave function is given by

$$
\boldsymbol{F}_{s, \boldsymbol{k}}=\frac{1}{\sqrt{2}}\left(\begin{array}{c}
\mathrm{e}^{\mathrm{i} \theta(\boldsymbol{k})} \\
s
\end{array}\right) \text {. }
$$

Figure 1 shows the dispersion relation and the density of states schematically.

\subsection{Long Wavelength Optical Phonon}

The long-wavelength optical phonons in the twodimensional graphite was discussed previously based on 
a valence-force-field model, ${ }^{14,15)}$ and in the following we shall limit ourselves to the long-wavelength limit. Let $\boldsymbol{u}=\left(u_{x}, u_{y}\right)$ be the relative displacement of two sub-lattice atoms $\mathrm{A}$ and $\mathrm{B}$, i.e.,

$$
\boldsymbol{u}(\boldsymbol{R})=\frac{1}{\sqrt{2}}\left[\boldsymbol{u}_{A}(\boldsymbol{R})-\boldsymbol{u}_{B}(\boldsymbol{R})\right]
$$

where $\boldsymbol{R}$ denotes a coordinate specifying a unit cell. In the long-wavelength limit $\boldsymbol{R}$ can be replaced by a continuous coordinate $\boldsymbol{r}$. Then we have

$$
\boldsymbol{u}(\boldsymbol{r})=\sum_{\boldsymbol{q}, \mu} \sqrt{\frac{\hbar}{2 N M \omega_{0}}}\left(b_{\boldsymbol{q} \mu}+b_{-\boldsymbol{q} \mu}^{\dagger}\right) \boldsymbol{e}_{\mu}(\boldsymbol{q}) \mathrm{e}^{\mathrm{i} \boldsymbol{q} \cdot \boldsymbol{r}}
$$

where $N$ is the number of unit cells, $M$ is the mass of a carbon atom, $\omega_{0}$ is the phonon frequency at the $\Gamma$ point, $\boldsymbol{q}=\left(q_{x}, q_{y}\right)$ is the wave vector, $\mu$ denotes the modes (t for transverse and 1 for longitudinal), and $b_{\boldsymbol{q} \mu}^{\dagger}$ and $b_{\boldsymbol{q} \mu}$ are the creation and destruction operators, respectively. Define

$$
q_{x}=q \cos \varphi(\boldsymbol{q}), \quad q_{y}=q \sin \varphi(\boldsymbol{q}),
$$

with $q=|\boldsymbol{q}|$. Then, we have

$$
\begin{aligned}
& \boldsymbol{e}_{\mathrm{l}}(\boldsymbol{q})=\mathrm{i}(\cos \varphi(\boldsymbol{q}), \sin \varphi(\boldsymbol{q})), \\
& \boldsymbol{e}_{\mathrm{t}}(\boldsymbol{q})=\mathrm{i}(-\sin \varphi(\boldsymbol{q}), \cos \varphi(\boldsymbol{q})) .
\end{aligned}
$$

The corresponding phonon Hamiltonian is written as

$$
\mathcal{H}_{\mathrm{ph}}=\sum_{\boldsymbol{q}, \mu} \hbar \omega_{0}\left(b_{\boldsymbol{q} \mu}^{\dagger} b_{\boldsymbol{q} \mu}+\frac{1}{2}\right)
$$

\subsection{Electron-Phonon Interaction}

The optical phonon modifies the distance between neighboring carbon atoms and therfore the band structure through the change in the resonance integral between carbon atoms. The corresponding effective Hamiltonian was obtained for acoustic phonons in ref. 14 and for optical phonons in ref. 15. The interaction Hamiltonian for the $\mathrm{K}$ point is given by

$$
\begin{aligned}
\mathcal{H}_{\mathrm{int}}^{\mathrm{K}} & =-\sqrt{2} \frac{\beta \gamma}{b^{2}}\left(\begin{array}{cc}
0 & u_{y}(\boldsymbol{r})+\mathrm{i} u_{x}(\boldsymbol{r}) \\
u_{y}(\boldsymbol{r})-\mathrm{i} u_{x}(\boldsymbol{r}) & 0
\end{array}\right) \\
& =-\sqrt{2} \frac{\beta \gamma}{b^{2}} \boldsymbol{\sigma} \times \boldsymbol{u}(\boldsymbol{r})
\end{aligned}
$$

and for the K' point

$$
\begin{aligned}
\mathcal{H}_{\mathrm{int}}^{\mathrm{K}^{\prime}} & =-\sqrt{2} \frac{\beta \gamma}{b^{2}}\left(\begin{array}{cc}
0 & u_{y}(\boldsymbol{r})-\mathrm{i} u_{x}(\boldsymbol{r}) \\
u_{y}(\boldsymbol{r})+\mathrm{i} u_{x}(\boldsymbol{r}) & 0
\end{array}\right) \\
& =-\sqrt{2} \frac{\beta \gamma}{b^{2}} \boldsymbol{\sigma}^{*} \times \boldsymbol{u}(\boldsymbol{r})
\end{aligned}
$$

where the vector product for vectors $\boldsymbol{a}=\left(a_{x}, a_{y}\right)$ and $\boldsymbol{b}=$ $\left(b_{x}, b_{y}\right)$ in two dimension is defined by $\boldsymbol{a} \times \boldsymbol{b}=a_{x} b_{y}-a_{y} b_{x}$ and $b=a / \sqrt{3}$ is the equilibrium bond length. The dimensionless parameter $\beta$ is given by

$$
\beta=-\frac{\mathrm{d} \ln \gamma_{0}}{\mathrm{~d} \ln b},
$$

where $\gamma_{0}$ is the resonance integral between nearest neighbor carbon atoms related to $\gamma$ through $\gamma=(\sqrt{3} a / 2) \gamma_{0}$.
This means that the lattice distortion gives rise to a shift in the origin of the wave vector or an effective vector potential, i.e., $u_{x}$ in the $y$ direction and $u_{y}$ in the $x$ direction. It should be noticed that this Hamiltonian is quite general because of the symmetry if the coupling parameter $\beta(\sim 2)$ is regarded as adjustable.

Explicitly, we have

$$
\mathcal{H}_{\mathrm{int}}=-\sqrt{\frac{\hbar}{2 N M \omega_{0}}} \sum_{\boldsymbol{q}, \mu} \sqrt{2} \frac{\beta \gamma}{b^{2}} V_{\mu}(\boldsymbol{q}) \mathrm{e}^{\mathrm{i} \boldsymbol{q} \cdot \boldsymbol{r}}\left(b_{\boldsymbol{q} \mu}+b_{-\boldsymbol{q} \mu}^{\dagger}\right),
$$

where

$$
V_{l}^{K}(\boldsymbol{q})=\left(\begin{array}{cc}
0 & -\mathrm{e}^{-\mathrm{i} \varphi(\boldsymbol{q})} \\
\mathrm{e}^{\mathrm{i} \varphi(\boldsymbol{q})} & 0
\end{array}\right)
$$

and

$$
V_{\mathrm{t}}^{K}(\boldsymbol{q})=\left(\begin{array}{cc}
0 & \mathrm{ie}-\mathrm{i} \varphi(\boldsymbol{q}) \\
\mathrm{ie} \mathrm{i}^{\mathrm{i} \varphi(\boldsymbol{q})} & 0
\end{array}\right)
$$

for the $\mathrm{K}$ point. For the $\mathrm{K}$ ' point, the corresponding quantities are obtained by the relation $V_{\mu}^{K^{\prime}}(\boldsymbol{q})=$ $V_{\mu}^{K}(-\boldsymbol{q})^{*}$. These matrices satisfy

$$
V_{\mu}(\boldsymbol{q})^{\dagger}=V_{\mu}(-\boldsymbol{q})
$$

\subsection{Phonon Green's Function}

The phonon thermal Green's function is defined by

$$
D_{\mu}(\boldsymbol{q}, \tau)=-\left\langle T_{\tau}\left[\phi_{\boldsymbol{q} \mu}(\tau) \phi_{\boldsymbol{q} \mu}(0)^{\dagger}\right]\right\rangle,
$$

with

$$
\phi_{\boldsymbol{q} \mu}=b_{\boldsymbol{q} \mu}+b_{-\boldsymbol{q} \mu} \dagger
$$

and

$$
\phi_{\boldsymbol{q} \mu}(\tau)=\exp (\tau \mathcal{H}) \phi_{\boldsymbol{q} \mu} \exp (-\tau \mathcal{H})
$$

where $\tau$ is the imaginary time and $T_{\tau}$ denotes the imaginary-time ordered product. The free Green's function is given by

$D_{\mu}^{0}\left(\boldsymbol{q}, \omega_{m}\right)=\int_{0}^{1 / k_{\mathrm{B}} T} \mathrm{e}^{\mathrm{i} \omega_{m} \tau} D_{\mu}^{0}(\boldsymbol{q}, \tau) d \tau=\frac{2 \hbar \omega_{0}}{\left(\mathrm{i} \omega_{m}\right)^{2}-\left(\hbar \omega_{0}\right)^{2}}$,

where the Matsubara frequency with integer $m$ is defined by $\omega_{m}=2 \pi k_{\mathrm{B}} T m$.

In general, $D\left(q, i \omega_{m}\right)$ is given by

$$
D_{\mu}\left(\boldsymbol{q}, \mathrm{i} \omega_{m}\right)=\frac{2 \hbar \omega_{0}}{\left(\mathrm{i} \omega_{m}\right)^{2}-\left(\hbar \omega_{0}\right)^{2}-2 \hbar \omega_{0} \Pi_{\mu}\left(\boldsymbol{q}, \mathrm{i} \omega_{m}\right)},
$$

with the self-energy $\Pi_{\mu}\left(\boldsymbol{q}, \mathrm{i} \omega_{m}\right)$. We shall consider the lowest order self-energy given by the diagram shown in Fig. 2. By making an analytic continuation $\mathrm{i} \omega_{m} \rightarrow \hbar \omega+\mathrm{i} 0$, we have the retarded Green's function

$$
D_{\mu}(\boldsymbol{q}, \omega)=\frac{2 \hbar \omega_{0}}{(\hbar \omega+\mathrm{i} 0)^{2}-\left(\hbar \omega_{0}\right)^{2}-2 \hbar \omega_{0} \Pi_{\mu}(\boldsymbol{q}, \omega)} .
$$

The phonon frequency is determined by the pole of $D_{\mu}(\boldsymbol{q}, \omega)$ as

$$
\left(\frac{\omega}{\omega_{0}}\right)^{2}-1=\frac{2}{\hbar \omega_{0}} \operatorname{Re} \Pi_{\mu}(\boldsymbol{q}, \omega) .
$$

As will become clear in the following, the phonon selfenergy is small. In this case, the shift of the phonon 
frequency is given by

$$
\Delta \omega_{\mu}=\frac{1}{\hbar} \operatorname{Re} \Pi_{\mu}\left(\boldsymbol{q}, \omega_{0}\right),
$$

and the broadening is given by

$$
\Gamma_{\mu}=-\frac{1}{\hbar} \operatorname{Im} \Pi_{\mu}\left(\boldsymbol{q}, \omega_{0}\right) .
$$

\section{$\S 3$. Optical-Phonon Self-Energy}

In the following we shall consider the phonons at the $\Gamma$ point, i.e., $|\boldsymbol{q}| \rightarrow 0$. Explicit matrix elements are

$$
\begin{aligned}
& \left|\left(s \boldsymbol{k}\left|V_{\mu}^{K}(\boldsymbol{q})\right| s^{\prime} \boldsymbol{k}\right)\right|^{2}=\left|\left(s \boldsymbol{k}\left|V_{\mu}^{K^{\prime}}(\boldsymbol{q})\right| s^{\prime} \boldsymbol{k}\right)\right|^{2} \\
& =\frac{1}{2}\left\{1-s s^{\prime} s_{\mu} \cos 2[\theta(\boldsymbol{k})-\varphi(\boldsymbol{q})]\right\}
\end{aligned}
$$

with $s_{\mu}=+1$ for the longitudinal mode and -1 for the transverse mode. The self-energy is given by

$$
\begin{aligned}
& \Pi_{\mu}(\boldsymbol{q}, \omega)=-g_{\mathrm{v}} g_{\mathrm{s}} L^{2} \sum_{s, s^{\prime}} \int \frac{\mathrm{d} \boldsymbol{k}}{(2 \pi)^{2}}\left(\frac{\beta \gamma}{b^{2}}\right)^{2} \frac{\hbar}{N M \omega_{0}} \\
& \times \frac{1}{2}\left\{1-s s^{\prime} s_{\mu} \cos 2[\theta(\boldsymbol{k})-\varphi(\boldsymbol{q})]\right\} \frac{f\left[\varepsilon_{s}(\boldsymbol{k})\right]-f\left[\varepsilon_{s^{\prime}}(\boldsymbol{k})\right]}{\hbar \omega-\varepsilon_{s}(\boldsymbol{k})+\varepsilon_{s^{\prime}}(\boldsymbol{k})+\mathrm{i} 0},
\end{aligned}
$$

where $f(\varepsilon)$ is the Fermi distribution function,

$$
f(\varepsilon)=\frac{1}{\mathrm{e}^{(\varepsilon-\zeta) / k_{\mathrm{B}} T}+1},
$$

with $\zeta$ being the chemical potential, and $g_{\mathrm{s}}$ and $g_{\mathrm{v}}$ are the spin and valley degeneracy $\left(g_{\mathrm{s}}=g_{\mathrm{v}}=2\right)$. The self-energy is independent of the modes after the angular integration over $\boldsymbol{k}$ and becomes

$$
\begin{aligned}
\Pi_{\mu}(\boldsymbol{q}, \omega) & =-g_{\mathrm{v}} g_{\mathrm{s}}\left(\frac{\beta \gamma}{b^{2}}\right)^{2} \frac{\hbar}{N M \omega_{0}} \frac{1}{2} L^{2} \sum_{s, s^{\prime}} \int \frac{\mathrm{d} \boldsymbol{k}}{(2 \pi)^{2}} \\
& \times \frac{f\left[\varepsilon_{s}(\boldsymbol{k})\right]-f\left[\varepsilon_{s^{\prime}}(\boldsymbol{k})\right]}{\hbar \omega-\varepsilon_{s}(\boldsymbol{k})+\varepsilon_{s^{\prime}}(\boldsymbol{k})+\mathrm{i} 0}
\end{aligned}
$$

It is clear in this equation that only interband processes $\left(s s^{\prime}=-1\right)$ contribute to the phonon self-energy, for which the matrix element squared is proportional to $1+s_{\mu} \cos [\theta(\boldsymbol{k})-\varphi(\boldsymbol{q})]$. Therefore, for the transverse mode $\left(s_{\mu}=-1\right)$ the interaction strength is maximum for an electron with $\boldsymbol{k}$ perpendicular to direction of $\pm \boldsymbol{q}$, i.e., with $\boldsymbol{k}$ in the direction of the displacement, and vanishes for $\boldsymbol{k}$ in the direction of $\pm \boldsymbol{q}$. For the longitudinal mode $\left(s_{\mu}=+1\right)$, on the other hand, the interaction is the strongest for $\boldsymbol{k}$ parallel to $\pm \boldsymbol{q}$ or the displacement. In spite of this anisotropic electron-phonon coupling, the resulting phonon dispersion becomes isotropic due to the isotropic dispersion in graphene. In carbon nanotubes, however, the wave vector along the circumference direction is quantized, which leads to softening of the longitudinal mode and hardening of the transverse mode in metallic carbon nanotubes. ${ }^{15)}$

The dependence of the interaction strength on the wave-vector direction can be understood by the $\boldsymbol{k} \cdot \boldsymbol{p}$ Hamiltonian (2.2) and the interaction Hamiltonian (2.15). In fact, the wave function with wave vector $\boldsymbol{k}$ is the eigen state of the pseudo-spin in the direction $\boldsymbol{k}$ and therefore only the part of the interaction Hamiltonian with the spin component perpendicular to the $\boldsymbol{k}$ direction, i.e., the displacement $\boldsymbol{u}$ in the $\boldsymbol{k}$ direction, contributes to interband transitions. Figure 3 shows the strength of interband transitions as a function of the direction of the wave vector measured from the direction of $\boldsymbol{u}$.

Define the dimensionless coupling parameter

$$
\lambda=\frac{g_{\mathrm{v}} g_{\mathrm{s}}}{4} \frac{36 \sqrt{3}}{\pi} \frac{\hbar^{2}}{2 M a^{2}} \frac{1}{\hbar \omega_{0}}\left(\frac{\beta}{2}\right)^{2}
$$

For $M=1.993 \times 10^{-23} \mathrm{~g}, a=2.46 \AA, \hbar \omega_{0}=0.196 \mathrm{eV}$, and $g_{\mathrm{v}}=g_{\mathrm{s}}=2$, we have

$$
\lambda \approx 3 \times 10^{-3} \frac{g_{\mathrm{v}} g_{\mathrm{s}}}{4}\left(\frac{\beta}{2}\right)^{2} .
$$

This parameter is slightly different from that defined in ref. 15. Then, we have

$$
\begin{aligned}
\Pi_{\mu}(\boldsymbol{q}, \omega) & =-\lambda \int_{0}^{\infty} \varepsilon \mathrm{d} \varepsilon[f(-\varepsilon)-f(\varepsilon)] \\
& \times\left(\frac{1}{\hbar \omega+2 \varepsilon+\mathrm{i} 0}-\frac{1}{\hbar \omega-2 \varepsilon+\mathrm{i} 0}\right),
\end{aligned}
$$

where use has been made of the density of states

$$
\int \frac{\mathrm{d} \boldsymbol{k}}{(2 \pi)^{2}} \delta[\varepsilon \pm \varepsilon(\boldsymbol{k})]=\frac{|\varepsilon|}{2 \pi \gamma^{2}} .
$$

We are calculating the self-energy of optical phonons starting with the known phonon modes in the twodimensional graphite. Therefore, the direct evaluation of the above self-energy causes a problem of double counting. ${ }^{20)}$ In fact, if we apply the above formula to the case of vanishing Fermi energy, we get the frequency shift due to virtual excitations of all electrons in the $\pi$ bands. However, this contribution is already included in the definition of the frequency $\omega_{0}$. In order to avoid such a problem, we have to subtract the contribution in the undoped graphene for $\omega=0$ corresponding to the adiabatic approximation.

This contribution can be obtained from the above by putting $\omega=0$ and $f(\varepsilon)=f_{0}(\varepsilon)$, with

$$
f_{0}(\varepsilon)= \begin{cases}0 & (\varepsilon>0) \\ 1 & (\varepsilon<0)\end{cases}
$$

as

$$
\Pi_{\mu}^{2 \mathrm{D}}(\boldsymbol{q}, \omega)=-\lambda \int_{0}^{\varepsilon_{\mathrm{c}}} \varepsilon \mathrm{d} \varepsilon\left(\frac{1}{2 \varepsilon+\mathrm{i} 0}+\frac{1}{2 \varepsilon-\mathrm{i} 0}\right)=-\lambda \varepsilon_{\mathrm{c}},
$$

where we have introduced the cutoff energy $\varepsilon_{\mathrm{c}}$ of the order of the half of the $\pi$-band width. Thus, the redefined self-energy becomes

$$
\begin{aligned}
\Pi_{\mu}(\boldsymbol{q}, \omega) & =\lambda \int_{0}^{\infty} \mathrm{d} \varepsilon[1-f(-\varepsilon)+f(\varepsilon)] \\
& +\frac{1}{2} \lambda \hbar \omega \int_{0}^{\infty} \mathrm{d} \varepsilon[f(-\varepsilon)-f(\varepsilon)] \\
& \times\left(\frac{1}{\hbar \omega+2 \varepsilon+\mathrm{i} 0}+\frac{1}{\hbar \omega-2 \varepsilon+\mathrm{i} 0}\right) .
\end{aligned}
$$


We have the relation

$$
f_{-\zeta}(\varepsilon)=1-f_{\zeta}(-\varepsilon)
$$

which leads to the conclusion that the self-energy is symmetric between $\zeta>0$ and $\zeta<0$, i.e., the electron-hole symmetry. In the following, therefore, we shall confine ourselves to the case $\zeta>0$. When we consider a level broadening effect by introducing a phenomenological imaginary part

$$
\delta=\frac{\hbar}{\tau}
$$

caused by scattering of electrons due to disorder, we have

$$
\begin{aligned}
\Pi_{\mu}(\boldsymbol{q}, \omega) & =\lambda \int_{0}^{\infty} \mathrm{d} \varepsilon[1-f(-\varepsilon)+f(\varepsilon)] \\
& +\frac{1}{2} \lambda(\hbar \omega+\mathrm{i} \delta) \int_{0}^{\infty} \mathrm{d} \varepsilon[f(-\varepsilon)-f(\varepsilon)] \\
& \times\left(\frac{1}{\hbar \omega+2 \varepsilon+\mathrm{i} \delta}+\frac{1}{\hbar \omega-2 \varepsilon+\mathrm{i} \delta}\right)
\end{aligned}
$$

At zero temperature, in particular, we have

$$
\Pi_{\mu}(\boldsymbol{q}, \omega)=\lambda \varepsilon_{\mathrm{F}}-\frac{1}{4} \lambda(\hbar \omega+\mathrm{i} \delta)\left(\ln \frac{\hbar \omega+2 \varepsilon_{\mathrm{F}}+\mathrm{i} \delta}{\hbar \omega-2 \varepsilon_{\mathrm{F}}+\mathrm{i} \delta}+\pi \mathrm{i}\right)
$$

where $\varepsilon_{\mathrm{F}}$ is the Fermi energy. In the clean limit $\delta \rightarrow 0$, we have

$\Pi_{\mu}(\boldsymbol{q}, \omega)=\lambda \varepsilon_{\mathrm{F}}-\frac{1}{4} \lambda \hbar \omega\left[\ln \left|\frac{\hbar \omega+2 \varepsilon_{\mathrm{F}}}{\hbar \omega-2 \varepsilon_{\mathrm{F}}}\right|+\pi \mathrm{i} \theta\left(\hbar \omega-2 \varepsilon_{\mathrm{F}}\right)\right]$.

The frequency shift, i.e., the real part, diverges logarithmically to $-\infty$ at $\varepsilon_{\mathrm{F}}=\hbar \omega_{0} / 2$. Apart from this logarithmic singularity, the phonon frequency increases roughly in proportion to $\varepsilon_{\mathrm{F}}$ for $\varepsilon_{\mathrm{F}}>\hbar \omega_{0}$. The broadening is nonzero only for $\varepsilon_{\mathrm{F}}<\hbar \omega_{0} / 2$.

Figure 4 shows the frequency shift and broadening for various values of $1 / \omega_{0} \tau$. For nonzero $\delta$ or $1 / \omega_{0} \tau$, the logarithmic singularity of the frequency shift and the sharp drop in the broadening disappear, but the corresponding features remain for $1 / \omega_{0} \tau \ll 1$.

\section{$\S 4$. Discussion}

The dependence of the frequency shift on the Fermi energy can be understood as the change in the effective force constant due to the reduction of screening due to interband transitions within the $\pi$ bands. For interband processes, the electron-phonon matrix element is independent of the electron-hole energy and therefore the contribution of each pair is proportional to the inverse of their energy due to the energy denominator. With the use of the fact that the density of states is proportional to the energy, this immediately leads to the contribution proportional to the $\pi$-band width $\varepsilon_{\mathrm{c}}$ for $\varepsilon_{\mathrm{F}}=0$. When the Fermi level increases, the states below the Fermi level in the conduction band can no longer contribute to the screening process and as a result the phonon frequency increases in proportion to $\varepsilon_{\mathrm{F}}$.

As shown in eqs. (3.1) and (3.2), the denominator vanishes for $\varepsilon(\boldsymbol{k})=\hbar \omega_{0} / 2$. This gives rise to the imaginary part of $\Pi$ as long as $\varepsilon_{\mathrm{F}}<\hbar \omega_{0} / 2$. For the real part, this causes the logarithmic divergence when the integral over $\varepsilon=\gamma|\boldsymbol{k}|$ is cut off at $\varepsilon=\hbar \omega_{0} / 2$, i.e., when $\varepsilon_{\mathrm{F}}=\hbar \omega_{0} / 2$. This is the origin of the behavior of the broadening and the energy shift shown in Fig. 4.

For the parameter $\gamma=6.46 \mathrm{eV} \cdot \AA$ giving $\gamma_{0}=$ $3.03 \mathrm{eV}$, the electron concentration corresponding to the condition $\varepsilon_{\mathrm{F}}=\hbar \omega_{0} / 2$ becomes $0.73 \times 10^{12} \mathrm{~cm}^{-2}$. For the mobility of $\mu \sim 10^{4} \mathrm{~cm}^{2} / \mathrm{Vs}$, the broadening $\hbar / \tau$ becomes $\sim 6 \mathrm{meV}$, where use has been made of $\mu=e v^{2} \tau / \varepsilon_{\mathrm{F}}$ with $v=\gamma / \hbar .^{21)}$ Actual broadening is likely to be larger than this estimated value because the transport relaxation time can be longer than the conventional relaxation time giving the broadening of the spectral function, giving $1 / \omega_{0} \tau>0.03$. It is possible, therefore, to observe the anomaly of optical phonons due to electron-phonon interactions.

\section{$\S 5$. Summary and Conclusion}

In this paper, the energy shift and broadening of optical phonons have been calculated as a function of the Fermi energy in a monolayer graphene. The broadening disappears suddenly when the Fermi energy exceeds the half of the optical-phonon energy in an undoped graphene sheet. The energy shift becomes minus infinity logarithmically when the Fermi energy becomes the half of the phonon energy and increases in proportion to the Fermi energy for sufficiently large Fermi energy. The logarithmic singularity is removed by level broadening due to disorder but the anomaly is expected to prevail in systems with high mobility.

\section{Acknowledgments}

The author thanks useful discussion with Mikito Koshino and Hidekatsu Suzuura. This work was supported in part by a 21st Century COE Program at Tokyo Tech "Nanometer-Scale Quantum Physics" and by Grant-in-Aid for Scientific Research from Ministry of Education, Culture, Sports, Science and Technology Japan.

\section{References}

1) J. W. McClure: Phys. Rev. 104 (1956) 666.

2) T. Ando: J. Phys. Soc. Jpn. 74 (2005) 777.

3) N. H. Shon and T. Ando: J. Phys. Soc. Jpn. 67 (1998) 2421.

4) Y. Zheng and T. Ando: Phys. Rev. B 65 (2002) 245420 .

5) T. Ando, Y. Zheng, and H. Suzuura: J. Phys. Soc. Jpn. 71 (2002) 1318.

6) T. Ando, A. B. Fowler, and F. Stern: Rev. Mod. Phys. 54 (1982) 437 and references cited therein.

7) K. S. Novoselov, A. K. Geim, S. V. Morozov, D. Jiang, Y. Zhang, S. V. Dubonos, I. V. Grigorieva, and A. A. Firsov: Science 306 (2004) 666.

8) K. S. Novoselov, A. K. Geim, S. V. Morozov, D. Jiang, M. I. Katsnelson, I. V. Grigorieva, S. V. Dubonos, and A. A. Firsov: Nature 438 (2005) 197.

9) Y. Zhang, Y.-W. Tan, H. L. Stormer, and P. Kim: Nature 438 (2005) 201.

10) A. C. Ferrari, J. C. Meyer, V. Scardaci, C. Casiraghi, M. Lazzeri, F. Mauri, S. Piscanec, D. Jiang, 
K. S. Novoselov, S. Roth, and A. K. Geim: condmat/0606284.

11) A. Gupta, G. Chena, P. Joshi, S. Tadigadapa, and P. C. Eklund: cond-mat/0606593.

12) S. Piscanec, M. Lazzeri, F. Mauri, A. C. Ferrari, and J. Robertson: Phys. Rev. Lett. 93 (2004) 185503.

13) M. Lazzeri, S. Piscanec, F. Mauri, A. C. Ferrari, and J. Robertson: Phys. Rev. B 73 (2006) 155426.

14) H. Suzuura and T. Ando: Phys. Rev. B 65 (2002) 235412 .

15) K. Ishikawa and T. Ando: J. Phys. Soc. Jpn. 75 (2006) 084713.

16) P. R. Wallace: Phys. Rev. 71 (1947) 622.

17) G. S. Painter and D. E. Ellis: Phys. Rev. B 1 (1970) 4747.

18) J. C. Slonczewski and P. R. Weiss: Phys. Rev. 109 (1958) 272.

19) H. Ajiki and T. Ando: J. Phys. Soc. Jpn. 62 (1993) 1255 .

20) N. A. Viet, H. Ajiki, and T. Ando: J. Phys. Soc. Jpn. 63 (1994) 3036.

21) T. Ando: J. Phys. Soc. Jpn. 75 (2006) 074716.

\section{Figure Captions}

Fig. 1 A schematic illustration of the dispersion relation $\varepsilon_{s}(\boldsymbol{k})$ and the density of states $D(\varepsilon)$ of the graphene. The vertical arrow indicates interband transitions due to electron-phonon interaction.

Fig. 2 A Feynman diagram for the self-energy for optical phonons with a wave vector $\boldsymbol{q}$ and Matsubara frequency $\omega_{m}$.

Fig. 3 The strength of interband transitions due to electron-phonon interaction as a function of the direction of the wave vector measured from the direction of the lattice displacement $\boldsymbol{u}$.

Fig. 4 The frequency shift (solid line) and broadening (dashed line) of optical phonons as a function of the Fermi energy. $\tau$ is a phenomenological relaxation time characterizing the level broadening effect due to disorder. 


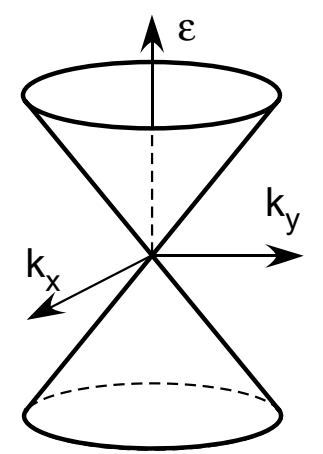

Fig. 1

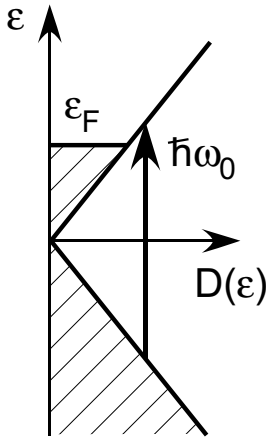

$\mathrm{D}(\varepsilon)$

$q, \omega_{m}$

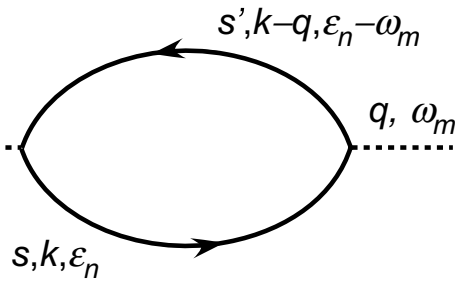

Fig. 2

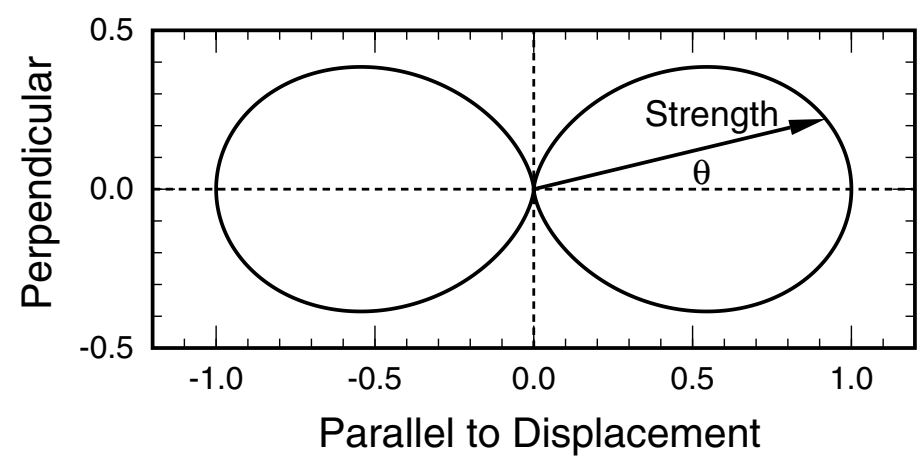

Fig. 3

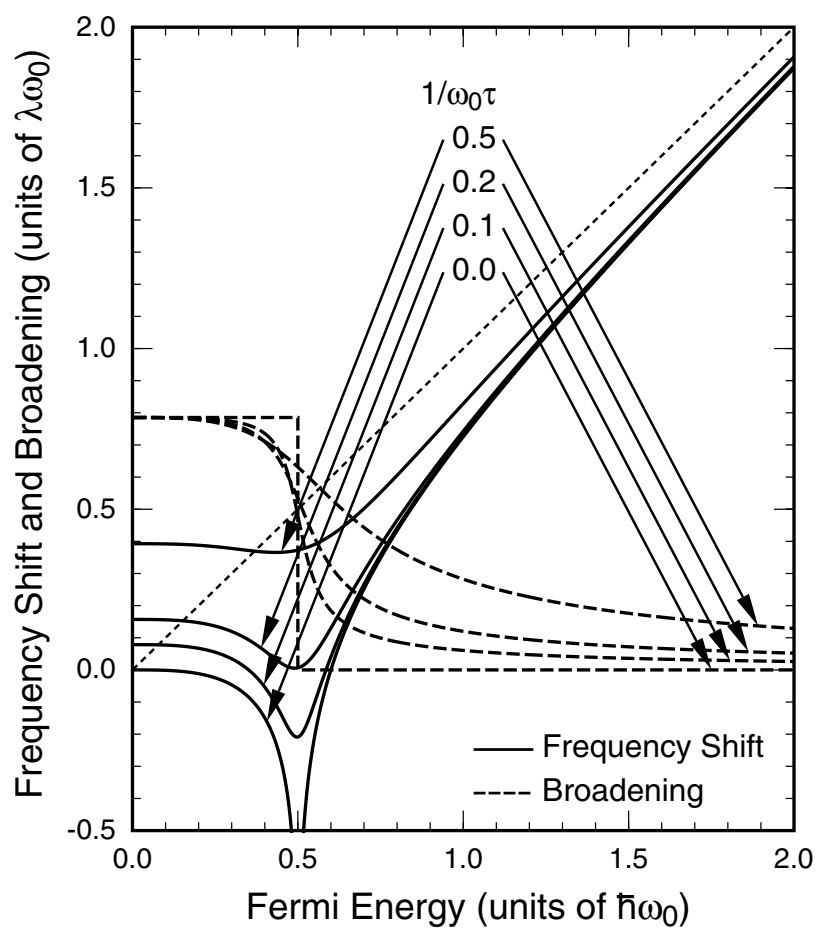

Fig. 4 